\title{
Escuelas con enfoque inclusivo, desde la perspectiva de integración: Familia, Alumnos y Docentes
}

\author{
Rocío Mabel Rotela Cáceres \\ lebamrossy.15@gmail.com
}

\author{
Nelly Isabel Rotela Cáceres \\ nellyisabelrotelacaceres@gmail.com \\ Facultad de Derecho, Ciencias Políticas y Sociales UNP
}

\section{RESUMEN}

En la actualidad en las escuelas se implementan los llamados planes de inclusión en los cuales se trata de integrar a los alumnos enfocándose en las distintas características de cada individuo. El estado se encuentra inmerso en un plan que no rinde fruto como se espera teniendo en cuenta que existen las leyes a nivel Nacional con sus reglamentaciones pero a la vez no se cuenta con una política pública que fomente su cumplimiento ya que no se cuenta con personal docente capacitado y esto conlleva a una falta de apoyo a alumnos con Necesidades Especiales Especiales (NEE). Teniendo en cuenta las informaciones recabadas podemos concluir que la inclusión es uno de los procesos más importantes en los últimos años en el campo educativo que repercute y condiciona ámbitos del desarrollo personal y condiciona el futuro profesional, laboral, cultural y social. La investigación se basó en la revisión bibliográfica de distintos artículos y materiales que hacían referencia a la investigación y objetivo planteado.

Palabras clave: Educación Inclusiva, diversidad, enfoque inclusivo, familia, alumnos, docentes. 


\title{
Schools with an inclusive approach from the perspective of integration: Family, Students and Teachers
}

\begin{abstract}
Currently schools are implementing the so-called inclusion plans in which they try to integrate students by focusing on the different characteristics of each individual. The state is immersed in a plan that does not bear fruit as expected, taking into account that there are laws at the national level with their regulations, but at the same time there is no public policy that encourages compliance since there are no personnel trained teacher and this leads to a lack of support for students with Special Special Needs (SEN). Taking into account the information collected, we can conclude that inclusion is one of the most important processes in recent years in the educational field that has repercussions and conditions areas of personal development and conditions the professional, labor, cultural and social future. The research was based on a bibliographic review of different articles and materials that made reference to the research and its stated objective.
\end{abstract}

Keywords: Inclusive Education, diversity, inclusive approach, family, students, teachers.

Artículo recibido: 19 enero 2020 Aceptado para publicación: 22 febrero 2020 Correspondencia: lebamrossy.15@gmail.com Conflictos de Interés: Ninguna que declarar 


\section{INTRODUCCIÓN}

La inclusión se refiere a la "identificación y minimización de las barreras para el aprendizaje y la participación, y maximización de los recursos para el apoyo de ambos procesos" (Ley 5136/13, Art. 3º. Inc. i).

La Constitución Nacional, la Ley Nº 1264/98 General de Educación, la Ley Nº 4995/13

De Educación Superior, la Ley No 5136/13 De Educación Inclusiva, con sus reglamentaciones, el Decreto Presidencial No 2837 del 22/12/2014 que reglamenta la Ley de Educación Inclusiva y la Resolución No 01/15 del Ministerio de Educación y Cultura por la cual se reglamenta el régimen de faltas y sanciones por incumplimiento de la Ley de Educación Inclusiva, constituyen el marco legal de la Educación inclusiva que se detalla brevemente.

\section{Constitución Nacional.}

En la Constitución Nacional de la República del Paraguay, sancionada el 20 de junio de 1992, en el Capítulo VII De la educación y de la cultura, Artículo 73 Del derecho a la educación y sus fines, se expresa que toda persona tiene derecho a la educación integral y permanente, que como sistema y proceso se realiza en el contexto de la cultura de la comunidad. Sus fines son el desarrollo pleno de la En el Artículo 74 Del derecho de aprender y de la libertad de enseñar, se garantiza la igualdad de oportunidades al acceso a los beneficios de la cultura humanística, ciencia y de la tecnología, sin discriminación alguna, también la libertad de enseñar, sin más requisitos que la idoneidad y la integridad ética (...).

Teniendo en cuenta lo que establece al respecto la Constitución Nacional, se debe velar desde las instituciones de educación superior, por esa igualdad de oportunidades en el acceso, sin discriminación alguna. Sin embargo, aún en la realidad, sobrevienen situaciones que denotan la necesidad de ir avanzando en la formación para la atención a las personas en situación de discapacidad, a fin de garantizar los derechos de los estudiantes, y la preparación adecuada del personal que tiene la obligación de brindar las oportunidades y contextos apropiados para la formación integral que se proclama personalidad humana y la promoción de la libertad y la paz, la justicia social, la solidaridad, la cooperación y la integración de los pueblos 


\section{Ley 5136/13 De Educación inclusiva.}

Entra en vigencia a partir del 22 de diciembre de 2013. Tiene por objeto establecer las acciones correspondientes para la creación de un modelo educativo inclusivo dentro del sistema regular, que remueva las barreras que limiten el aprendizaje y la participación, facilitando la accesibilidad de los alumnos con necesidades específicas de apoyo educativo por medio de recursos humanos calificados, tecnologías adaptativas y un diseño universal.

En el Paraguay, el 23 de diciembre del año 2013 se promulgó la Ley 5136/13 de Educación Inclusiva, y a través de la misma se pretende establecer acciones para la creación de un modelo educativo inclusivo dentro del sistema regular, que remueva las barreras que limiten el aprendizaje y la participación, facilitando la accesibilidad de los alumnos con necesidades específicas de apoyo educativo por medio de recursos humanos calificados, tecnologías adaptativas y un diseño universal (Artículo $1^{\circ}$ ).

Según la publicación del MEC denominada "La Educación Inclusiva en la Nueva Escuela Pública Paraguaya. Marco referencial, normativo y operativo" (2011), se introduce la definición basada en Ainscow, Booth y Dyson (2006): La Educación inclusiva hace referencia a un proceso sistemático de mejora e innovación educativa para promover la presencia, el rendimiento y la participación de todo el alumnado en la vida escolar de los centros donde son escolarizados, con particular atención a aquellos alumnos o alumnas más vulnerables a la exclusión, el fracaso escolar o la marginación, detectando y eliminando, para ello, las barreras que limitan dicho proceso (p. 25).

La denominación de necesidades educativas especiales, está referida a los alumnos con dificultades importantes en el aprendizaje, esta definición, intenta crear un enfoque en el que se acentúen las necesidades pedagógicas que estos presentan y los recursos que se han de proporcionar, en lugar de realizar categorías diagnósticas por el tipo de discapacidad que los afectan, si bien no se deben obviar los aspectos clínicos en la evaluación e intervención de estas necesidades. Las necesidades educativas especiales pueden ser temporales y permanentes y a su vez pueden ser debidas a causas:

- Físicas

- Psíquicas

- Situación socio-familiar

- Otros casos de inadaptación (cultural, lingüística, etc.) 
La educación inclusiva es el modelo que busca atender las necesidades de aprendizaje de todos los niños, niñas, jóvenes y adultos con especial énfasis en aquellos que son vulnerables a la marginalidad y la exclusión social.

La educación inclusiva es un proceso de que toda la sociedad debe vivir, ya que es el punto de partida para normalizar la educación de todos los alumnos y alumnas y del mismo modo brindar diversas oportunidades para el desarrollo de las personas que tienen o viven con discapacidad o marginación.

La educación inclusiva beneficia a todos los estudiantes. Les permite desarrollar fortalezas y dones individuales, con expectativas altas y apropiadas, y trabajar en objetivos individuales mientras participan en la vida de la clase con otros estudiantes.

La inclusión fomenta una cultura escolar de respeto y pertenencia, y brinda oportunidades para aprender y aceptar las diferencias individuales, disminuyendo el impacto del acoso y la intimidación. Permite el desarrollo de la amistad con una amplia variedad de niños, cada uno con sus propias necesidades y habilidades individuales.

R.Feuerestein y K. Frankestein citado por Batalla (2000) dicen:

"la escuela debe brindarle al niño todo aquello que el medio social donde vive le niega" entonces aquí se hace evidente que el marco contenedor es afectivo y va de la mano con innumerables estrategias que aquí no vienen al caso. Es necesario saber que no todos los niños presentarán problemas solo porque provienen de este marco institucional, deberemos establecer a través del hacer educativo cotidiano quienes son los que evidencian dificultades (P.8).

Según LUS, María Angélica: "La educación de los niños con necesidades educativas especiales es una tarea compartida por padres de familia y profesionales. Una actitud positiva y participativa de parte de los padres de familia propicia la integración educativa y social".

Para GONZÁLEZ, Eugenia. "Los padres son los principales asociados en lo tocante a las necesidades educativas especiales de sus hijos y a ellos debería corresponder en la medida de lo posible la elección del tipo de educación que desean sea impartida a sus hijos".

En tanto que para GARCÍA, Cediño Ismael "Se define al niño con necesidades educativas especiales, como aquél que tiene dificultades significativas mayores que las 
del resto del grupo para aprender, y que requiere de apoyos o servicios que habitualmente no se encuentran disponibles en las escuelas regulares"

Para Alfonso (2010): "un alumno/a con necesidades educativas especiales es aquel/aquella que requiera, en un periodo de su escolarización o a lo largo de toda ella, determinados apoyos y atenciones educativas específicas por padecer discapacidades físicas, psíquicas, sensoriales o por manifestar graves trastornos de personalidad o conducta.” (p.2)

Así, trabajar de modo conjunto, dentro de la escuela y con las familias y otros actores de la comunidad, facilita que la escuela pueda mejorar la educación de los alumnos, al tiempo que se promocione un reconocimiento mutuo entre familias y profesorado. Como dicen los teóricos del capital social, si no hay redes de participación, las posibilidades de la acción colectiva son escasas (Bolívar, 2006). Pag.140 revista de Educación.

La familia como cualquier otro agente tiene un papel clave en el desarrollo de los niños y jóvenes; nadie puede ni debe encargarse de su educación en exclusiva ni en solitario, todos deben analizar y determinar cuál es su función en el proceso educativo, con el fin de contribuir al desarrollo integral del alumnado dando una respuesta educativa de calidad. La sociedad y la escuela han evolucionado, pero no sólo ellas, sino que la familia también ha sufrido cambios sustanciales: desde una configuración patriarcal o matriarcal a una familia nuclear; de una escasa participación hasta su papel activo y protagonista en la escuela y en las dinámicas internas y educativas relacionadas con sus hijos.

"La inclusión es una idea bonita, interesante, pero todavía es una idea", es decir se está produciendo un cambio a nivel teórico que todavía está demasiado lejos de verse plasmado en la práctica. Como otro profesor comentó, "para dar un paso más es necesaria una legislación que facilite las pautas para que la inclusión no se deba a la buena voluntad de los profesionales de un centro, sino a unas leyes que refuercen que eso sea así”. La importancia de mantener una comunicación clara y sincera con los padres. Para fomentar su colaboración es fundamental implicarles en actividades motivadoras y no solo citarles cuándo se presenta algún problema "Hay que plantearles lo bueno que tiene su hijo, que tiene muchas cosas, y ayudarles a superar sus miedos. Les ayuda la afectividad que reciben sus hijos y verlos contentos". Otras estrategias que 
comentan para fomentar la implicación de las familias es el impartir charlas cada trimestre, hacer jornadas de puertas abiertas y fomentar una relación de confianza. Como propuestas de mejora, sugieren bajar las ratios y poder crear agrupaciones flexibles, contar con la presencia diaria del orientador y tener en cuenta la diversidad del alumnado a la hora de elaborar los proyectos educativos. Una educación de calidad debe lograr el nivel máximo de cada persona, por lo tanto tiene que ser individualizada y ofrecer las adaptaciones necesarias que cada persona necesite. Debe permitir acceder a un trabajo en el futuro. Tiene que estar orientada a la vida en la comunidad y ser útil para que puedan desenvolverse en su día a día. Una educación de calidad tiene que ayudarles a ser felices. (Verdugo y Rodríguez-Aguilella, 2008)

El Conjunto de Materiales de la UNESCO (2004) para la Formación de Profesores: Las Necesidades Especiales en el Aula, ofrece una variedad de estrategias muy útiles para desarrollar recursos para las escuelas. Igualmente, útil es el Índice para la Inclusión porque estimula a las escuelas a revisar la forma en que responden a la diversidad del alumnado" (p.35). El Índice, en particular, define "apoyo" como todo aquello que facilita que la escuela sea capaz de responder a la diversidad. Esta definición podría servir para disuadir a aquellos docentes u otras personas que piensan que el apoyo siempre implica recursos adicionales o que están fuera del aula ordinaria (Booth et al., 2000, p.55).

Según la UNESCO (2000) La primera tarea para establecer apoyos efectivos consiste en movilizar aquellos recursos que ya existen, tanto dentro como fuera de las escuelas. En muchos casos esto será lo único que hay que hacer para atender las necesidades de una amplia gama de estudiantes. No es posible decidir qué apoyos adicionales son necesarios a menos que se empleen al máximo los recursos ya disponibles en las escuelas (p.42).

Según el Foro Mundial Sobre Educación (2000), el tipo de estrategias que se usan comúnmente para aumentar esta efectividad incluyen:

- Apoyo entre pares, donde los estudiantes trabajan cooperativamente dentro de la clase y son capaces de apoyarse mutuamente con su trabajo

- Apoyo de maestro a maestro, donde el personal docente de una escuela trabaja de manera colaborativa para decidir la mejor forma de atender las diversas necesidades de sus estudiantes 
- Llevar a cabo una "diferenciación” en el aula en la que los maestros desarrollan la capacidad y los recursos necesarios para gestionar diferentes tipos o niveles de actividad que respondan a la diversidad de estudiantes que tienen en sus aulas

- Aulas de recursos donde se puedan ofrecer recursos especializados, donde los maestros especialistas (si hay disponibilidad) puedan trabajar y donde se pueda ofrecer a los estudiantes enseñanza especializada, siempre y cuando la necesiten.

Según Fernández, Montero, Martínez, Orcasitas y Villaescusa, (2015) Las familias de las personas con discapacidad han ido ocupando muy diferentes posiciones a lo largo del tiempo. Los profundos cambios que han experimentado tanto la comprensión como las prácticas sociales sobre la discapacidad han acompañado, de forma paralela, a un aumento de la valoración de las familias como contexto esencial para el desarrollo y la calidad de vida. El hecho de reconocer a las personas con discapacidad como sujetos de derechos con capacidad de interlocución ha contribuido a empoderar a las familias y a enfatizar una mayor implicación. Turnbull (2003) resume los diferentes roles que las familias han jugado en este proceso, pasando de ser objeto de intervenciones psicoterapéuticas o formativas a ser sujetos activos en la determinación de necesidades y la toma de decisiones. Aunque inicialmente el estudio de las familias se centraba en enfoques más patológicos (Córdoba, Gómez y Verdugo, 2008), en estos momentos se adoptan enfoques holísticos que toman como referencia unificada el concepto de Calidad de Vida Familiar y consideran la visión de la propia familia un elemento esencial (Summers et al., 2005).

La Calidad de Vida se ha convertido en una de las principales referencias en el ámbito de la discapacidad, funcionando como principio orientador de la práctica y convirtiéndose, en consecuencia, en un referente para la evaluación de los servicios y programas. La incorporación de la idea de calidad de vida a las familias es más reciente (inicio de la década de 2000) y, como señalan algunos autores, requiere de un mayor desarrollo teórico (Wang y Kober, 2011). A la hora de definir la Calidad de Vida Familiar, una de las definiciones más aceptadas (Verdugo, Rodríguez y Sainz, 2012) es la propuesta por Park et al. (2003) que la caracterizan como el grado en el que se colman las necesidades de los miembros de la familia, en el que disfrutan de su tiempo junto y en el que puedan hacer cosas que son importantes para ellos. 
La familia como red social primaria es esencial en cualquier etapa de la vida; gracias a ella, el ser humano hace valiosa y significativa su vida.

La escuela tiene distintas funciones como la de transmitir a las nuevas generaciones conocimientos que han sido adquiridos paulatinamente de generación en generación; buscar la inclusión en la educación; fortalecer las aptitudes naturales para desarrollarla y contribuir a la formación de la personalidad de los alumnos., como institución social encargada de llevar a cabo la educación en forma organizada, apoyada por planes y programas de estudios impartidos en diferentes niveles. Además, la escuela es una institución social encargada de llevar a cabo la educación en forma organizada, mediante el apoyo de planes y programas de estudio impartidos en los diferentes niveles, para desarrollar habilidades y destrezas, pero principalmente para inculcarles valores humanos a los alumnos que de alguna manera orientarán su vida y despertar y mantener su interés por elevar su nivel cultural.

En consecuencia, la familia juega un papel importante en este sentido, para la inclusión escolar y se sitúa en segundo plano con funciones sociales diferentes y ante todo tanto la familia como la escuela han de unir esfuerzos para lograr superar las grandes dificultades.

La propuesta que presentamos se fundamenta en los pilares de la educación para el futuro: aprender a conocer, aprender a ser, aprender a hacer y aprender a vivir en comunidad.

Además, una escuela de calidad tiene en cuenta que la familia es uno de los principales recursos de apoyo para lograr progresos en los aprendizajes de los estudiantes y, por tanto, la invita a participar e involucrarse en la toma de decisiones educativas con respecto a sus hijos e hijas, construyendo con ella una relación positiva y de colaboración.

Los fundamentos en los que se basa el desarrollo de una escuela inclusiva están centrados en el tipo de educación que en la sociedad queremos ofrecer a nuestras futuras generaciones.

Se podría igualar a esa idea que como madre o padre tenemos sobre la educación que queremos para nuestras hijas e hijos, una educación en la que les tengan en cuenta como personas; en la que aprendan a ser, a hacer, a aprender y a convivir (UNESCO, 1996); 
en la que disfruten de momentos de enseñanza y aprendizaje de calidad; y en la que las relaciones se entablen en base al respeto, la equidad y la justicia social.

Una educación inclusiva tiene como objetivo lograr una educación de calidad, que se caracteriza por las dos cualidades que cualquier sistema y centro educativo deben cuidar como son la excelencia y la equidad. La excelencia en la educación se define por estar centrada en alcanzar los más altos niveles de desarrollo de cada alumna y alumno para que puedan participar como ciudadanas y ciudadanos con plenos derechos y deberes. La educación debe para ello cumplir con tres funciones: preparación para el futuro, preparar en las competencias que van a ser necesarias para ser personas ciudadanas en el futuro; rescate de la base cultural del pasado, que haga al alumnado conocedor de aquellos saberes, valores y cultura que nos sustentan, y que les permitirá proyectarse en el futuro; preparación para vivir el presente, que se reconozcan sus necesidades y demandas actuales y que se les aporte con las herramientas para vivir su vida plenamente en el momento que les toca vivir.

\section{La trayectoria de la educación inclusiva, marco internacional}

Los fundamentos en los que se basa el desarrollo de una escuela inclusiva están centrados en el tipo de educación que en la sociedad queremos ofrecer a nuestras futuras generaciones.

Se podría igualar a esa idea que como madre o padre tenemos sobre la educación que queremos para nuestras hijas e hijos, una educación en la que les tengan en cuenta como personas; en la que aprendan a ser, a hacer, a aprender y a convivir (UNESCO, 1996); en la que disfruten de experiencias de enseñanza y aprendizaje de calidad; y en la que las relaciones se entablen en base al respeto, la equidad y la justicia social.

Según el Informe a la UNESCO de la Comisión Internacional sobre la Educación para el Siglo XXI presidida por Jaques Delors, «La educación encierra un tesoro» (UNESCO, 1996), la educación es el proceso de promoción de un aprendizaje social que se basa en cuatro pilares: «aprender a conocer, aprender a hacer, aprender a vivir juntos y aprender a ser», con el objetivo de desarrollar ciudadanos y ciudadanas que contribuyan a la construcción de sociedades modernas democráticas y promover la cultura de paz. Estos cuatro pilares, y en particular el relativo a aprender a convivir, son parte central de los valores que deben guiar el desarrollo de una educación inclusiva. 
Cabe reconocer el protagonismo que durante la última década del Siglo XX y el comienzo del XXI ha tenido la UNESCO en su labor para poner la Educación Inclusiva en la agenda política y en los sistemas educativos de la mayoría de los países del mundo. Estos cuatro pilares que se formalizan con el Informe Delors ya son parte de los principios fundamentales de la Conferencia Mundial de «Educación para Todos» que se llevó a cabo en Jomtien, Tailandia en 1990 (UNESCO, 1994). En dicha conferencia, representantes de los países participantes se comprometieron a que, en el espacio de una década, garantizarían la respuesta a las necesidades básicas de aprendizaje de cualquier niña o niño, joven, o persona adulta. En aquella ocasión, el foco

No obstante, en 1994, la UNESCO, junto con el Ministerio de Educación y Ciencia de España, consideró necesaria organizar una nueva Conferencia Mundial en Salamanca para profundizar en el compromiso sobre el derecho a una educación de calidad para todas las personas, particularmente aquellas con necesidades educativas especiales, e la conferencia se centró particularmente en el acceso a la educación para todas las personas.

Para Tomé, J. M. este tema se ha enmarcado en la idea de que la escuela debe ser una institución inclusiva y solidaria con el fin de responder tanto a las necesidades de cada una de las personas cuanto a las de la construcción de una sociedad no discriminadora, sino justa e igualitaria. Es este, sin duda, un desafío que exige la comprensión de un cúmulo de problemas complejos así como coraje y capacidad de gestión para enfrentar las dificultades que se presentan en la implementación de soluciones a la vez eficaces y éticas, dado que se oponen a prejuicios y prácticas instaladas e, igualmente, porque son no solamente respetuosas sino también inspiradoras de las diferencias.

Se exige pues, a la escuela, que sea inclusiva e integradora en la realidad, más allá de la retórica pedagógica, haciendo de ella una comunidad de práctica del aprendizaje, abierta a todos, en la que los alumnos desarrollan un trabajo colaborativo y los profesores conforman equipos colaborativo

Según la declaración de Salamanca (1999): La educación de los niños con necesidades educativas especiales es una tarea compartida por padres y profesionales. Una actitud positiva de los padres propicia la integración escolar y social. Los padres de un niño con necesidades educativas especiales necesitan apoyo para asumir sus responsabilidades. La función de las familias y los padres podría mejorarse facilitando la información 
necesaria simple y clara. Se deberán estrechar las relaciones de cooperación y de apoyo entre los administradores de las escuelas, los profesores y los padres. Se procurará que estos últimos participen en la adopción de decisiones en actividades educativas en el hogar y en la escuela. (Pag 3 - 18)

\section{ESTRATEGIAS METODOLÓGICAS O MATERIALES Y MÉTODOS}

La metodología utilizada en este artículo tuvo un enfoque cualitativo con base en una revisión documental, el cual utilizó investigaciones que hacen referencia al contexto de Educación Inclusiva. Teniendo en cuenta el objetivo que se plantea y buscando la comprensión del mismo este método nos permite tener analizar los mismos desde el punto de vista de estudios previos realizados, permitiéndonos interiorizarnos sobre el tema. Se utilizó la información de publicaciones en textos tanto nacionales como internacionales, con el fin de obtener de manera organizada los estudios y aportes sobre el tema.

Se realizó la revisión de los distintos documentos teniendo en cuenta los criterios necesarios para dar respuesta a la investigación, al ser seleccionados y analizados los distintos artículos e informaciones que contribuían a nuestra investigación se procedió a la descarga de los mismos. Se procedió a señalar los aportes y resultados de cada material a fin de destacar los hallazgos.

\section{RESULTADOS Y DISCUSIÓN}

MEL, Ainscow afirma que "La mayoría de las personas aprenden mejor con otras personas. Aparte del estímulo intelectual que ello supone, está también la confianza que nos da el apoyo y la ayuda de los demás mientras trabajamos"

El concepto de "Inclusión" está relacionado, con la naturaleza misma de la educación general y de la escuela común.

La inclusión implica que todos los niños, niñas y adolescentes de una determinada comunidad aprendan juntos, independientemente de sus condiciones personales, sociales o culturales.

Al mismo tiempo, la escuela es un espacio preferencial para desarrollar actitudes individuales y sociales que generen cambios significativos entre las personas. La escuela inclusiva está sustentada en una pedagogía que se opone a la selección jerárquica y propone la voluntad de desarrollar a los alumnos en un contexto escolar respetuoso de la diversidad personal y colectiva. 
Cuanto más inclusivas sean las escuelas comunes en su funcionamiento, menos adolescentes quedarán fuera sea de los espacios recreativos (parques, plazas, clubes), comunitarios (grupos religiosos, de organización vecinal,) o de la escuela (programas de educación no formal, formal, otros espacios educativos).

Por tanto, no será necesario integrarlos: creemos que si trabajamos en este abordaje en las diferentes etapas y con un enfoque inclusivo, la repitencia y el fracaso escolar, flagelo de nuestra época, se minimizará algún día.

En la actualidad ya no se tiende a definir a los "alumnos especiales" desde sus características individuales (dificultades de inteligencia, audición, visión, motricidad, etcétera), sino desde la respuesta que les debe dar el medio ambiente. Se cambia así el nombre de discapacitado sensorial, mental, etc., por el de alumno con necesidades educativas especiales (NEE). Con esta nomenclatura, se intenta poner de relieve el compromiso social y educativo frente a estos alumnos y alumnas. Se considera que el etiquetamiento y la rotulación son modalidades de discriminación negativa, basadas en esa habitual predisposición humana de intolerancia frente a las diferencias individuales.

Esta enfoque /nominación intenta mirar desde otra perspectiva el problema de las NEE: dejar a un lado la perspectiva de los alumnos con "sus dificultades individuales" para profundizar en el análisis de la propuesta educativa que se les brinda.

Aunque este concepto está actualmente en discusión, es importante que lo entendamos bien ya que es una terminología presente en nuestra Ley General de educación, en el Código de niñez y adolescencia (aunque allí también se hace referencia a niños y niñas con capacidades diferentes) pero, en síntesis, las características del concepto de Necesidades Educativas Especiales (NEE) son:

- Son un continuo, que van desde características generales a otras más específicas.

- Están identificadas con la necessidade de ayudas pedagógicas concretas permite el logro de los fines de la Educación.

- Las NEE están vinculadas a las características del sujeto y al contexto en que se desenvuelve su vida.

- El enfoque educativo ligado al concepto de NEE asegura que mediante una intervención pedagógica adecuada pueden mejorar las condiciones de aprendizaje y de vida de los sujetos. 


\section{CONCLUSIÓN O CONSIDERACIONES FINALES}

Teniendo en cuenta las informaciones recabadas podemos concluir que la inclusión es uno de los procesos más importantes en los últimos años en el campo educativo que repercute y condiciona ámbitos del desarrollo personal y condiciona el futuro profesional, laboral, cultural y social. Es por ello que el sistema educativo no solo depende de las instituciones del estado sino que también es relevante la participación de los distintos estamentos esto incluye a los docentes, alumnos y a la família, siendo esta última de vital importância para lograr los objetivos de una integración completa ya que el acompañamiento y el apoyo constante es un fator que redunda en el cumplimiento de las normativas. El Ministerio de Educación y Ciencias cuenta con las Leyes y las reglamentaciones en cuanto a Educación Inclusiva se refiere pero no tiene el acompañamiento de una Política Pública que es necesaria para que se pueda aplicar, teniendo en cuenta que los docentes tienen que ser capacitados para asi poder llevar adelante la integración de los alumnos con NEE. Esto teniendo en cuenta el respeto a los derechos fundamentales tales como el derecho a la igualdad de oportunidades, a la accesibilidad universal y a una educación de calidad, lo que implica por una parte, la ausencia de discriminación y, por otra, la adopción de medidas positivas a tal fin. Por ello la Educación inclusiva al estar reglamentada, es aún un gran desafío la puesta en práctica, si no se trabaja articuladamente con todos los organismos de implementación para velar por el cumplimiento de los derechos de los ciudadanos. En este sentido cabe remarcar que este derecho constituye una exigencia social, un reto para la sociedad, para los propios individuos, y de forma especial, para los docentes y sus instituciones. Una prioridad ha de ser por tanto la formación y preparación de los docentes, a fin de responder de forma ajustada a las variadas situaciones de inclusividad que plantean los alumnos y, especialmente, los más vulnerables.

\section{LISTA DE REFERENCIAS}

Alfonso Naranjo, B. (2010). Alumnos/as con NEE, familia y escuela, juntos por la integración. Revista digital para profesionales de la enseñanza, (9), 1-11. https://feandalucia.ccoo.es/docu/p5sd7361.pdf

ARNAIZ SÁNCHEZ, PILAR. (2003). Educación Inclusiva: una escuela para todos. Ed. Aljibe.

Atención a la Diversidad- MEC Paraguay- Organización de Estados Iberoamericanos para la educación la ciencia y la cultura rescatado por web: (Microsoft Word - 
DEVALLE, Alicia y VEGA, Viviana. La diversidad y la formacil363n docente.doc) (terras.edu.ar)

BATALlA, M. (2000). Animación a la lectura. Recuperado de http://alerce.pntic.mec.es/ rloo0004/infantil/articulos.htm

Bolívar, A. (2006). Familia y escuela: dos mundos llamados a trabajar en común. Revista de Educación, 39, 119-146.

CASANO, R., Y LEZCANO, F. (2012). Educación en la escuela inclusiva. Buenos Aires: Editorial Magisterio del Río de La Plata.

CASTRO, J. (S7a). Educación Especial Necesidades Educativas Especiales. Observatorio de la Discapacidad Instituto de Mayores y Servicios Sociales. Recuperado de Observatorio de la Discapacidad Instituto de Mayores y Servicios Sociales

Educacion inclusiva.pdf (lugareditorial.com.ar)

ESCUELA INCLUSIVA- TEORIA PRACTICAS DE ENSEÑANZA EN LAS ESCUELAS PRIMARIAS- AUTOR Jose Maria Tome pag. 7

Fernández, A., Montero, D., Martínez, N., Orcasitas, J. R. y Villaescusa, M. (2015). Calidad de vida familiar: marco de referencia, evaluación e intervención. Siglo Cero, 46(2), 254, 7-29.

GARCÍA, Cediño Ismael. "Necesidades educativas especiales". En: El desarrollo de la integración educativa en el aula regular. México, 1999. SEP. P. 20

GONZÁLEZ, Eugenia. "Necesidades educativas especiales". Intervención psicoeducativa. Editorial C.C.S. p. 58.

http://www.edu.xunta.gal/centros/cafi/aulavirtual/pluginfile.php/16068/mod_resource/conte nt/0/PILAR_ARNAIZSANCHEZ/EducacionInclusiva_PilarArnaiz.pdf

https://lugareditorial.com.ar/descargas/libros/Educacion\%20inclusiva.pdf

https://sid.usal.es/idocs/F8/ART10955/valoracion_inclusion_educativa.pdf

LUS, María Angélica. De la integración escolar integradora. Buenos Aires. Editorial Paidos. p. 160.

Ministerio de Educación y Cultura (2011). La Educación Inclusiva en la Nueva Escuela Pública Paraguaya: Marco referencial, normativo y operativo. 
Ministerio de Educación y Cultura (2011). Plan Nacional de Educación 2024. Hacia el centenario de la Escuela Nueva de Ramón Indalecio Cardozo.

UNESCO (2004). Temario abierto de educación inclusiva. Santiago de Chile. Recuperado de http://sid.usal.es/idocs/F8/FDO23200/temario_abierto_educacion_inclusiva.pdf

UNESCO (2004). Temario Abierto sobre Educación Inclusiva. Santiago: OREALC / UNESCO. Pag.15, 19,20, 60

Verdugo, M. A. y Rodríguez-Aguilella, A. (2008). Valoración de la inclusión educativa desde diferentes perspectivas. Siglo Cero, 39(4), 5-25.

Declaración de Salamanca de principios políticos y prácticos para las necesidades educativas especiales. Cuadernillo de Integración educativa No. 3. 1999. p. 3-18.

MEL, Ainscow. "Reconsideraciones de las necesidades educativas especiales". Necesidades educativas especiales asociadas a la discapacidad. UPN. SEP. p. 248 